OPEN ACCESS

Edited by:

Alessandro Vitale,

University Hospital of Padua, Italy

Reviewed by:

Marco Massani,

ULSS2 Marca Trevigiana, Italy

Tingfan Wu,

GE Healthcare, China

${ }^{*}$ Correspondence:

Xianjun Zhou

18661809968@163.com

Qian Dong

18661801885@163.com

${ }^{t}$ These authors have contributed equally to this work

Specialty section: This article was submitted to

Surgical Oncology,

a section of the journal

Frontiers in Oncology

Received: 21 July 2021 Accepted: 04 February 2022

Published: 07 March 2022

Citation:

He Y, Hu B, Zhu C, XuW, Ge Y, Hao X, Dong $B$, Chen $X$, Dong $Q$ and Zhou $X$ (2022) A Novel Multimodal Radiomics

Model for Predicting Prognosis of Resected Hepatocellular Carcinoma.

Front. Oncol. 12:745258.

doi: 10.3389/fonc.2022.745258

\section{A Novel Multimodal Radiomics Model for Predicting Prognosis of Resected Hepatocellular Carcinoma}

\author{
Ying $\mathrm{He}^{1 \dagger}$, Bin $\mathrm{Hu}^{2 \dagger}$, Chengzhan $\mathrm{Zhu}^{3}$, Wenjian $\mathrm{Xu}^{2}$, Yaqiong $\mathrm{Ge}^{4}$, Xiwei $\mathrm{Hao}^{1}$, \\ Bingzi Dong ${ }^{5}$, Xin Chen ${ }^{1}$, Qian Dong ${ }^{1,5,6 *}$ and Xianjun Zhou ${ }^{1,5^{*}}$ \\ ${ }^{1}$ Department of Pediatric Surgery, The Affiliated Hospital of Qingdao University, Qingdao, China, ${ }^{2}$ Department of Radiology, \\ The Affiliated Hospital of Qingdao University, Qingdao, China, ${ }^{3}$ Department of Hepatobiliary and Pancreatic Surgery, The \\ Affiliated Hospital of Qingdao University, Qingdao, China, ${ }^{4}$ GE Healthcare, Shanghai, China, ${ }^{5}$ Shandong Key Laboratory of \\ Digital Medicine and Computer Assisted Surgery, The Affiliated Hospital of Qingdao University, Qingdao, China, ${ }^{6}$ Shandong \\ College Collaborative Innovation Center of Digital Medicine Clinical Treatment and Nutrition Health, Qingdao University, \\ Qingdao, China
}

Objective: To explore a new model to predict the prognosis of liver cancer based on MRI and CT imaging data.

Methods: A retrospective study of 103 patients with histologically proven hepatocellular carcinoma (HCC) was conducted. Patients were randomly divided into training $(n=73)$ and validation $(n=30)$ groups. A total of 1,217 radiomics features were extracted from regions of interest on CT and MR images of each patient. Univariate Cox regression, Spearman's correlation analysis, Pearson's correlation analysis, and least absolute shrinkage and selection operator Cox analysis were used for feature selection in the training set, multivariate Cox proportional risk models were established to predict diseasefree survival (DFS) and overall survival (OS), and the models were validated using validation cohort data. Multimodal radiomics scores, integrating CT and MRI data, were applied, together with clinical risk factors, to construct nomograms for individualized survival assessment, and calibration curves were used to evaluate model consistency. Harrell's concordance index ( $\mathrm{C}$-index) values were calculated to evaluate the prediction performance of the models.

Results: The radiomics score established using CT and MR data was an independent predictor of prognosis (DFS and OS) in patients with HCC $(p<0.05)$. Prediction models illustrated by nomograms for predicting prognosis in liver cancer were established. Integrated CT and MRI and clinical multimodal data had the best predictive performance in the training and validation cohorts for both DFS [(C-index $(95 \% \mathrm{Cl})$ : $0.858(0.811-0.905)$ and 0.704 (0.563-0.845), respectively)] and OS [C-index (95\% Cl): $0.893(0.846-0.940)$ and $0.738(0.575-0.901)$, respectively]. The calibration curve showed that the multimodal radiomics model provides greater clinical benefits. 


\begin{abstract}
Conclusion: Multimodal (MRI/CT) radiomics models can serve as effective visual tools for predicting prognosis in patients with liver cancer. This approach has great potential to improve treatment decisions when applied for preoperative prediction in patients with HCC.
\end{abstract}

Keywords: liver cancer, multimodal imaging, computed tomography, MRI, radiomics, nomogram

\section{INTRODUCTION}

Hepatocellular carcinoma (HCC) is the most common primary liver tumor, accounting for $75 \%-85 \%$ of liver cancers (1). HCC is the second most common cause of cancer death worldwide and has high morbidity and mortality rates (2). Surgical resection and local ablation remain the most commonly used radical treatment methods for HCC; however, tumors recur in $70 \%$ of cases after hepatectomy and $25 \%$ of cases after liver transplantation, and the 5 -year overall survival (OS) rate is only approximately $25 \%-55 \%$ (3-5). Hence, patients with HCC have a poor prognosis after surgery, and the high disease recurrence rate represents a great challenge to successful treatment $(3,6)$. Therefore, the identification of reliable predictors of early recurrence is critical for patient risk stratification, support for treatment decisions, and improvement of long-term survival.

At present, relevant tumor factors, such as lesion diameter, cirrhosis, multifocality, poorly differentiated tumor, and microvascular invasion (MVI), are recognized as risk factors for early disease recurrence (7-10); however, most of these features can only be evaluated by postoperative histopathological examination, which is invasive, and the results are prone to a missed diagnosis. In oncology, the application of radiomics, which involves the transformation of traditional medical images into high-dimensional, quantitative, and exploitable imaging data, enables in-depth characterization of tumor phenotypes and has the potential to provide information on intra-tumor heterogeneity and predict posttreatment survival $(11,12)$. Multimodal machine learning is a method to process and interpret multimodal information through machine learning. Multimodal fusion is used to fuse multimodal information and perform targeted prediction classification or regression problems (13-15). Medical imaging can include data in different forms, such as CT, MRI, PET, ultrasound, and X-rays. In different guidelines, either CT or MRI is proposed as the best imaging modality for the diagnosis of

\footnotetext{
Abbreviations: HCC, hepatocellular carcinoma; DFS, disease-free survival; OS, overall survival; C-index, Harrell's concordance index; TACE, transarterial chemoembolization; ALT, alanine aminotransferase; AST, aspartate aminotransferase; TBIL, total bilirubin; ALB, albumin; AFP, alpha-fetoprotein; ROI, region of interest; ICCs, the intra-class coefficient and the inter-class correlation coefficient; GLCM, gray-level co-occurrence features matrix-based features; GLRLM, gray-level run-length matrix-based features; GLSZM, gray-level size zone matrix-based features; GLDM, gray-level dependence matrix-based features; Log, Laplace wavelet; LASSO, least absolute shrinkage and selection operator; KM, Kaplan-Meier; Radscore, radiomics score; MVI, microvascular invasion; BMI, body mass index; PV_TT, portal vein tumor thrombosis; PLT, platelet count; HBsAg, hepatitis B surface antigen status; PT, prothrombin time; NEUT, neutrophil count.
}

HCC (16-18). Recent HCC management guidelines recognize an increasing role for gadoxetic acid-enhanced MRI in early diagnosis and monitoring post-resection (19). CT or MRI can all confirm the diagnosis if a nodule larger than $1-\mathrm{cm}$ diameter is found with typical vascular features of HCC (hypervascularity in the arterial phase with washout in the portal venous or delayed phase) (20). Further, both CT and MR functional scans can be useful as supplements to conventional plain scan and dynamic enhancement to improve the accuracy of follow-up evaluation of liver cancer (21). In recent years, several qualitative MRI and CT imaging features have been reported. Preliminary evidence suggests that radiomics features have the potential to predict OS and tumor recurrence in patients with HCC, for example, by assessing peritumor parenchymal enhancement, satellite nodules, and non-smooth tumor margins, which are noninvasive predictors of early HCC recurrence (22-24).

Multimodal fusion technology can be divided into pixel level, feature level, and decision level, which are used to fuse abstract features and decision results in original data (13-15). To date, radiomics has been successfully applied in the study of nasopharyngeal carcinoma, non-small cell lung cancer, and rectal cancer (25-27), demonstrating the great potential for the development of this approach; however, to our knowledge, the use of contrast analysis of CT-enhanced sequence and MRenhanced sequence data to assess patient prognosis remains rare. In this study, we combined these two novel imaging techniques and explored the performance of multimodal radiomics models derived from $\mathrm{MR}$ and $\mathrm{CT}$ image data for prognostic evaluation following HCC resection.

\section{MATERIALS AND METHODS}

\section{Patients}

This study was approved by the Ethics Committee of the Affiliated Hospital of Qingdao University. Due to its retrospective nature, the need for patient written informed consent was waived. From February 2014 to December 2020, we collected information from 306 patients with liver cancer, and 135 patients with primary HCC were recruited, based on the following inclusion criteria: 1) pathologically confirmed liver cancer recorded in the medical records at our hospital and 2) CT and MRI examinations performed within the previous 2 weeks before hepatectomy. The exclusion criteria were as follows: 1) other preoperative treatments [transarterial chemoembolization (TACE)], targeted drugs, and radiofrequency ablation), except hepatectomy $(\mathrm{n}=11) ; 2)$ incomplete clinicopathological report $(\mathrm{n}=10) ; 3)$ CT image and MR image quality was poor, and the 
lesion could not be recognized or the lesion image was less than three layers $(\mathrm{n}=3)$; 4$)$ lost to follow-up $(\mathrm{n}=4)$; and 5$)$ error occurred in the feature extraction process $(n=4)$. The final study population included 103 patients. The entire cohort was randomly divided into a training cohort $(\mathrm{n}=73)$ and a validation cohort $(n=30)$ (ratio, 7:3). Training queues were used to build single-modal and multimodal radiomics models, which were evaluated using validation queues.

\section{Clinical Endpoints and Follow-Up}

The endpoints of this study were disease-free survival (DFS) and OS. DFS was measured from the date of surgery until disease progression, death from any cause, or the last visit in follow-up (censored), and nomograms were also built based on the DFS. Disease progression, including local recurrence distant metastasis, was confirmed by clinical examination and imaging methods such as abdominopelvic CT or MRI or was biopsyproven. OS was defined as the time to death from any cause. All patients were followed up after surgery. Serum alanine transaminase (ALT), aspartate transaminase (AST), total bilirubin (TBIL), albumin (ALB), and alpha-fetoprotein (AFP) levels were obtained. Liver ultrasound examination was performed monthly within the 3 months after surgery and once every 3 months thereafter. CT examination of the lungs and enhanced CT or MRI of the liver were performed every 3 months during the first 2 years and once every 6 months thereafter. The minimum follow-up period was 3 days after surgery, while the maximum follow-up time was 92.8 months.

\section{Image Acquisition \\ CT Scanning Methods and Parameters}

Three-stage enhanced scans of the upper abdomen were obtained using a German CT (SOMATOM Definition Flash, Siemens, Munich, Germany) and an American Discovery CT (GE Healthcare, Chicago, IL, USA). Scans ranged from the top of the liver to the lower edges of both kidneys. Scanning parameters were as follows: voltage, $120 \mathrm{kV}$; current, 200-350 mA; scanning layer thickness, $5 \mathrm{~mm}$; layer spacing, $5 \mathrm{~mm}$; and matrix, $512 \times$ 512. For contrast-enhanced scanning, a double-barreled highpressure syringe was used to inject iohexol, containing $350 \mathrm{mg} /$ $\mathrm{ml}$ of iodine, via the peripheral vein (flow rate, $3.0 \mathrm{ml} / \mathrm{s}$; dose, 1.5 $\mathrm{ml} / \mathrm{kg}$ ). The delay times for the arterial, venous, and equilibrium phases were 30,60 , and 120 s, respectively.

\section{MRI Scanning Methods and Parameters}

MRI scanning was conducted using a 3.0 T Signa HDXT MR superconducting apparatus and an 8-channel body-phase front coil. Rapid volume acquisition Liver Acquisition with Volume Acceleration (LAVA) imaging of the liver was conducted using the following parameters: repetition time (TR), $4.2 \mathrm{~ms}$; echo time (TE), $2.0 \mathrm{~ms}$; layer thickness, 4.8-5.4 mm; layer spacing, 1.4-2.7 $\mathrm{mm}$; field, $42.0 \times 33.6 \mathrm{~cm}$; and matrix, $320 \times 192$. The contrast agent, gadolinium diethylenetriamine penta-acetic acid, was used for enhanced scanning (dose, $0.2 \mathrm{mmol} / \mathrm{kg}$; injection flow rate, 2.0 $\mathrm{ml} / \mathrm{s}$ ). The delay times of the arterial, portal, and equilibrium phases were 20-23, 60, and $180 \mathrm{~s}$, respectively.

\section{Tumor Segmentation}

The tumor region of interest (ROI) was manually delineated on multi-phase CT and MR images by a radiologist with more than 10 years of experience (Reader 1) using ITK-SNAP (version 3.6.0; http://www.itksnap.org) to segment each tumor CT stage and MR stage. A two-dimensional ROI of the largest section of the tumor was selected, outlined, and saved as an NII file. Two weeks later, Reader 1 randomly selected 50 HCC patients and delineated the ROI again to evaluate the intra-class correlation coefficient of ROI. Additionally, another radiologist (Reader 2) independently performed ROI mapping for the randomly selected $50 \mathrm{HCC}$ patients to evaluate the inter-class correlation coefficient.

\section{Image Preprocessing and Feature Extraction}

At the beginning of extraction, pre-processing was necessary to improve discrimination between texture features. To eliminate the batch effect of different equipment, all the data were normalized through $\mathrm{Z}$-score standardization to a standard intensity range with a mean value of 0 and $\mathrm{SD}$ of 1 , and the image slices were resampled to voxel size $=1 * 1 * 1 \mathrm{~cm}^{3}$. With the use of IBSI compliant AK software (Analysis Kit Software, version 3.3.0, GE Healthcare), 1,217 radiomics features were extracted from CT and MR images, including first-order statistical features, morphological features, gray-level cooccurrence features, matrix-based features (GLCM), gray-level run-length matrix-based features (GLRLM), gray-level size zone matrix-based features (GLSZM), gray-level dependence matrixbased features (GLDM), and (Log) Laplace wavelet changes. Furthermore, intra-class and inter-class correlation coefficients (ICCs) were used to evaluate the intra-observer and interobserver reproducibility of feature extraction. The intra-class correlation coefficient was calculated by comparing the ROI of Reader 1 twice. The inter-class correlation coefficient between the groups was evaluated by comparing the ROI of Reader 1 with that of Reader 2. When ICCs exceeded 0.75 both within and between observers, this feature was considered to have a good consistency. Finally, the ICC range for CT (Balance, Venous, and Artery) was $0.175-1$, and 917 features with ICC $>0.75$ were retained for each phase. The ICC range for MR (Balance, Venous, and Artery) was $0.256-1$, and 946 features with ICC > 0.75 were retained.

\section{Feature Selection and Model Construction}

Features with ICC values $>0.75$ both within and between groups were retained for further analysis. In the training set, features with $p<0.05$ in univariate Cox regression analysis were retained, and Spearman's correlation analysis and Pearson's correlation analysis were applied to eliminate characteristics that were highly correlated (selected coefficient threshold $|\mathrm{r}|=0.8$ ). The least absolute shrinkage and selection operator (LASSO) Cox regression with 10 -fold cross-validation was used for further feature screening. Then, features with non-zero coefficients selected by LASSO analysis were linearly weighted. Next, radiomics scores (Radscores) were calculated for each patient. 
The Radscore was the result of the Cox regression radiomics model. It was the linear combination weighted by the corresponding LASSO coefficients of each feature selected of each patient, and patients were then divided into high-risk and low-risk groups, according to their best truncation value in each model and the labeled high-risk group (riskscore $=1$ ) and the low-risk group (riskscore $=0$ ). Kaplan-Meier $(\mathrm{KM})$ analysis was used to plot DFS and OS curves, and the log-rank test was used to evaluate the differences between high-risk and low-risk groups. The same threshold was then applied to the validation queue. Cindex values were used to evaluate the performance of the model.

\section{Nomogram Construction}

First, univariate Cox analysis was used to analyze risk factors and screen for features with $p<0.05$. Clinical factors with $p<0.05$ and Radscore for CT and MRI data combined (Combined_radscore) were included in the multivariate Cox stepwise regression model, to investigate independent predictors of survival in HCC patients. Clinical factors and Combined_radscore (with $p<0.05$ ) in the univariate Cox analysis were enrolled to establish a nomogram to predict patients' 2-year, 4-year, and 5-year survival rates. C-index values were used to evaluate the performance of the model, and calibration curves were generated and discrimination ability was quantified to compare predicted and actual survival rates.

\section{Statistical Analysis}

All statistical analyses were performed using R3.5.1 (https://www. r-project.org/). A t-test or Mann-Whitney $U$ test was used to evaluate differences in continuous variables, and the chi-square or Fisher's exact test to assess differences in categorical variables. Continuous numerical variables are represented by the median (25th percentile, 75 th percentile), and categorical variables are represented by percentages. Shapiro's test function in the $\mathrm{R}$ package was used to test for normality. Spearman's correlation analysis and Pearson's correlation analysis were used to eliminate redundant features. Pearson's correlation analysis was used for the features that conform to the normal distribution, and Spearman's correlation analysis was used for the features that do not conform to normal distribution. The surv_cutpoint function in the $\mathrm{R}$ package was used to calculate optimal truncation values. The KM method and log-rank test were used to estimate DFS and OS. Calibration curves were used to evaluate the degree of alignment of nomograms. Two-sided $p$-values $<0.05$ were considered significant.

\section{RESULTS}

\section{Patient Characteristics}

Patient demographics and clinicopathological features are presented in Table 1. Of the 103 patients included in the study, $83(80.6 \%)$ were male, and the median age of all patients was $57.0(32.0-73.0)$ years. There were no statistically significant differences in clinicopathological factors between patients in the training $(\mathrm{n}=73,70 \%)$ and validation $(\mathrm{n}=30$, $30 \%$ ) cohorts ( $p=0.558-0.997)$. A total of 44 patients had death endpoints. The median values for DFS and OS of the total patient group $(\mathrm{n}=103)$ were $25.9(0.1-88.1)$ months and $43.7(0.1-92.8)$ months, respectively.

\section{Radiomics Signature Construction}

Features retained after each feature dimension reduction are listed in Supplementary Table S1. Finally, for prediction of DFS, 7, 12, and 17 features were selected from CT, MRI, and their combined features, respectively, and used to build models. For prediction of OS, 8,16 , and 17 features were selected to establish the model from CT, MRI, and their combined features, respectively. The details of selected features of DFS and OS are included in Supplementary Figure S1 and Table S2. The calculated CT_radscore, MRI_radscore, and Combined_radscore were based on selected features.

We performed the univariate Cox analysis to determine the role of clinical features of patients on DFS in HCC (Table 2). Three clinical characteristics, namely, tumor diameter, liver capsule invasion, and MVI were identified by univariate analysis $(p<0.05)$. Clinical features with $p<0.05$ were included in backward stepwise multivariate regression analysis. The results show that MVI was an independent predictor of HCC in the multivariable analysis $(p<0.05)$. We performed the univariate Cox analysis to determine the role of clinical characteristics on the OS of patients in HCC (Table 3). Six clinical characteristics, namely, body mass index (BMI), tumor diameter, MVI, portal vein tumor thrombosis (PV_TT), platelet count (PLT), and Bleeding_volume were identified by univariate analysis $(p<0.05)$. Clinical characteristics with $p<0.05$ were included in backward stepwise multivariate regression analysis. The results show that BMI, MVI, and Bleeding_volume were independent predictors of HCC in the multivariable analysis $(p<$ 0.05). The clinical models were built based on clinical risk features, and the Clinical_score of each model was calculated.

Combined_radscore and clinical factors were included in univariate Cox regression for analyzing DFS, and factors with $p<0.05$ were included in backward stepwise multivariate Cox regression analysis (Table 4). The results show that Radscore and MVI were independent predictors of HCC in the multivariable analysis $(p<0.05)$. Combined_radscore and clinical factors were included in univariate Cox regression for analyzing OS, and factors with $p<0.05$ were included in backward stepwise multivariate Cox regression analysis (Table 5). The results show that Radscore, MVI, PLT, and Bleeding_volume were independent predictors of HCC in the multivariable analysis $(p<0.05)$. CT+MRI_Clinical Model was established based on significant clinical risk features and Radscore. CT+MRI+Clinical_score of the models were calculated.

CT_radscore, MRI_radscore, Combined_radscore, Clinical_score, and CT+MRI+Clinical_score were divided into a high-risk group and a low-risk group according to the optimal cutoff value of each group, and then DFS and OS KM curves were plotted. KM curves methods and log-rank test estimating DFS (Figure 1) in the training cohort showed that patients in the lowrisk group had significantly better outcomes than those in the high-risk group (all log-rank $p<0.05$ ) using the model. We then performed the same analyses in the validation cohort. Each model had similar results in the validation cohort $(p<0.05)$. 
TABLE 1 | Demographic and clinicopathological characteristics of patients with liver cancer.

\begin{tabular}{|c|c|c|c|c|}
\hline Variable & & Training cohort $(\mathrm{N}=73)$ & Validation cohort $(\mathbf{N}=30)$ & $p$ \\
\hline \multirow[t]{2}{*}{ Age (years), } & $>60$ & $32(0.44)$ & $12(0.40)$ & 0.721 \\
\hline & $\leq 60$ & $41(0.56)$ & $18(0.60)$ & \\
\hline \multirow[t]{2}{*}{ Gender } & Male & $60(0.82)$ & $7(0.23)$ & 0.520 \\
\hline & Female & $13(0.18)$ & $23(0.77)$ & \\
\hline \multirow[t]{2}{*}{ Alcohol abuse (\%) } & Present & $13(0.18)$ & $6(0.20)$ & 0.794 \\
\hline & Absent & $60(0.82)$ & $24(0.80)$ & \\
\hline \multirow[t]{2}{*}{$\operatorname{AFP}(\mathrm{ng} / \mathrm{ml}, \%)$} & $\leq 20$ & $32(0.44)$ & $11(0.37)$ & 0.503 \\
\hline & $>20$ & $41(0.56)$ & $19(0.63)$ & \\
\hline \multirow[t]{2}{*}{ HBV (\%) } & Present & $63(0.86)$ & $23(0.77)$ & 0.231 \\
\hline & Absent & $10(0.14)$ & $7(0.23)$ & \\
\hline \multirow[t]{2}{*}{ HBsAg (\%) } & Positive & $62(0.85)$ & $23(0.77)$ & 0.316 \\
\hline & Negative & $11(0.15)$ & $7(0.23)$ & \\
\hline \multirow[t]{2}{*}{ Pos_operation_TACE (\%) } & Present & $29(0.40)$ & $10(0.33)$ & 0.543 \\
\hline & Absent & $44(0.60)$ & $20(0.67)$ & \\
\hline \multirow[t]{2}{*}{ Tumor diameter (cm, \%) } & $\leq 5 \mathrm{~cm}$ & $52(0.71)$ & $17(0.57)$ & 0.153 \\
\hline & $>5 \mathrm{~cm}$ & $21(0.29)$ & $13(0.43)$ & \\
\hline \multirow[t]{2}{*}{ Tumor number (\%) } & $\geq 2$ & $8(0.11)$ & $3(0.10)$ & 0.835 \\
\hline & $<2$ & $65(0.89)$ & $27(0.9)$ & \\
\hline \multirow[t]{2}{*}{ MVI (\%) } & Present & $35(0.48)$ & $17(0.57)$ & 0.421 \\
\hline & Absent & $38(0.52)$ & $13(0.43)$ & \\
\hline \multirow[t]{2}{*}{ PV-TT (\%) } & Present & $3(0.04)$ & $2(0.07)$ & 0.627 \\
\hline & Absent & $70(0.96)$ & $28(0.93)$ & \\
\hline \multirow[t]{2}{*}{ Satellite lesions (\%) } & Present & $9(0.12)$ & $3(0.10)$ & 0.997 \\
\hline & Absent & $64(0.88)$ & $27(0.90)$ & \\
\hline \multirow[t]{2}{*}{ Liver cirrhosis (\%) } & Present & $61(0.84)$ & $26(0.87)$ & 0.924 \\
\hline & Absent & $12(0.16)$ & $4(0.13)$ & \\
\hline \multirow[t]{2}{*}{ Surgical margin (\%) } & $<1 \mathrm{~cm}$ & $26(0.36)$ & $16(0.53)$ & 0.094 \\
\hline & $\geq 1 \mathrm{~cm}$ & $47(0.64)$ & $14(0.47)$ & \\
\hline \multirow[t]{2}{*}{ Liver capsule invasion (\%) } & Present & $39(0.53)$ & $13(0.43)$ & 0.352 \\
\hline & Absent & $34(0.47)$ & $17(0.57)$ & \\
\hline \multirow[t]{2}{*}{ Surgical approach (\%) } & Laparoscopy & $22(0.30)$ & $10(0.33)$ & 0.750 \\
\hline & Non-laparoscopy & $51(0.70)$ & $20(0.67)$ & \\
\hline \multirow[t]{2}{*}{ Histopathological grading } & I, II & $41(0.56)$ & $16(0.53)$ & 0.793 \\
\hline & III, IV & $32(0.44)$ & $14(0.47)$ & \\
\hline \multirow[t]{2}{*}{ Child-Pugh score (\%) } & A & $71(0.97)$ & $26(0.87)$ & 0.058 \\
\hline & $\mathrm{B}$ & $2(0.03)$ & $4(0.13)$ & \\
\hline \multirow[t]{2}{*}{ CNLC (\%) } & I, || & $66(0.90)$ & $25(0.83)$ & 0.309 \\
\hline & III, IV & $7(0.10)$ & $5(0.17)$ & \\
\hline \multirow[t]{2}{*}{ Bleeding_volume (ml, \%) } & $\leq 400$ & $64(0.88)$ & $27(0.90)$ & 0.997 \\
\hline & $>400$ & $9(0.12)$ & $3(0.10)$ & \\
\hline $\mathrm{BMI}\left(\mathrm{kg} / \mathrm{m}^{2}\right)$ & & $25.28(22.67,26.57)$ & $23.81(21.87,25.69)$ & 0.209 \\
\hline ALT (IU/L) & & $38(21,69)$ & $40.50(26.50,97.93)$ & 0.408 \\
\hline AST (IU/L) & & $29(21,57)$ & $31.5(22.25,64,35)$ & 0.452 \\
\hline TBIL ( $\mu \mathrm{mol} / \mathrm{L})$ & & 17.07 (13.56-22.50) & $18.31(13.61,25.65)$ & 0.338 \\
\hline ALB (g/L) & & $40.05(37.29,43.41)$ & $40.71(37.25,43.96)$ & 0.836 \\
\hline PT (s) & & $10.5(9.80,11.10)$ & $10.60(9.83,11.17)$ & 0.825 \\
\hline $\operatorname{PLT}\left(10^{9} / \mathrm{L}\right)$ & & $160(127,209)$ & $164(116,190)$ & 0.554 \\
\hline NEUT $\left(10^{9} / \mathrm{L}\right)$ & & $2.97(2.12,4.74)$ & $3.51(2.88,4.52)$ & 0.200 \\
\hline Lymphocyte $\left(10^{9} / \mathrm{L}\right)$ & & $1.9(1.36,3.77)$ & $1.71(1.43,2.57)$ & 0.862 \\
\hline
\end{tabular}

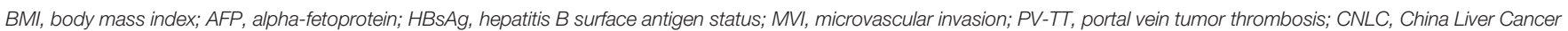
Staging; ALT, alanine aminotransferase; AST, aspartate aminotransferase; TBIL, total bilirubin; ALB, albumin; PT, prothrombin time; PLT, platelet count; NEUT, neutrophil count.

KM curves methods and log-rank test estimating OS (Figure 2) in the training cohort showed that patients in the low-risk group had significantly better outcomes than those in the high-risk group $(p<0.05)$. We then performed the same analyses in the validation cohort, and similar results were observed.

\section{Development and Assessment of a Radiomics Nomogram}

To provide the clinician with a quantitative method to predict patients' probability of 2-year, 4-year, and 5-year DFS and OS and to demonstrate the incremental value of the radiomics signature for individualized assessment of DFS and OS, both radiomics nomograms were built in the training cohort (Figures 3A, B).

For prediction of DFS, Radscore, tumor diameter, liver capsule invasion, and MVI were finally retained to establish a nomogram for DFS prediction (Figure 3A), and BMI, tumor diameter, PV_TT, PLT, Bleeding_volume, and Radscore were retained for use in establishing the prognostic prediction nomogram for OS (Figure 3B). The performance of each 
TABLE 2 | Univariate and multivariate analyses of training cohort to identify patient clinical features with prognostic value for DFS.

\begin{tabular}{|c|c|c|c|c|}
\hline \multirow[t]{2}{*}{ Variable } & \multicolumn{2}{|c|}{ Univariate analysis } & \multicolumn{2}{|c|}{ Multivariate analysis } \\
\hline & HR (95\% Cl) & $p$-Value & $\mathrm{HR}(95 \% \mathrm{Cl})$ & $p$-Value \\
\hline Age & $0.994(0.961-1.028)$ & 0.708 & & \\
\hline Gender & $1.712(0.723-4.055)$ & 0.222 & & \\
\hline BMI & $1.014(0.992-1.036)$ & 0.229 & & \\
\hline Alcohol & $1.088(0.506-2.342)$ & 0.829 & & \\
\hline Liver cirrhosis & 1.436 (0.607-3.399) & 0.410 & & \\
\hline Histopathological grade & 1.361 (0.842-2.199) & 0.209 & & \\
\hline Tumor diameter & $1.128(1.02-1.247)$ & $<0.05$ & $1.07(0.96-1.19)$ & 0.244 \\
\hline Liver capsule invasion & 1.907 (1.036-3.509) & $<0.05$ & $1.41(0.74-2.72)$ & 0.299 \\
\hline Surgical margin & 1.025 (0.963-1.091) & 0.445 & & \\
\hline Tumor number & $1.329(0.583-3.027)$ & 0.499 & & \\
\hline Satellite lesions & 1.43 (0.602-3.393) & 0.418 & & \\
\hline $\mathrm{MVI}$ & $4.338(2.31-8.147)$ & $<0.05$ & $3.95(2.07-7.54)$ & $<0.05$ \\
\hline$P V_{-} T T$ & $1.412(0.34-5.867)$ & 0.635 & & \\
\hline HBV & $0.833(0.352-1.971)$ & 0.677 & & \\
\hline HBsAg & 0.999 (0.997-1.003) & 0.953 & & \\
\hline Surgical approach & $1.198(0.626-2.291)$ & 0.585 & & \\
\hline Pos_operation_TACE & $1.652(0.911-2.996)$ & 0.099 & & \\
\hline AFP & $1.000(0.999-1.000)$ & 0.547 & & \\
\hline PLT & 0.999 (0.995-1.004) & 0.806 & & \\
\hline PT & $1.002(0.989-1.015)$ & 0.749 & & \\
\hline Alb & $1.014(0.949-1.084)$ & 0.675 & & \\
\hline TBIL & 0.954 (0.907-1.003) & 0.067 & & \\
\hline ALT & 1.001 (0.998-1.003) & 0.594 & & \\
\hline AST & 1.001 (0.999-1.003) & 0.307 & & \\
\hline NEUT & 1.088 (0.978-1.209) & 0.120 & & \\
\hline Lymphocyte & 0.987 (0.96-1.016) & 0.379 & & \\
\hline Bleeding_volume & 1.000 (0.999-1.000) & 0.201 & & \\
\hline Child-Pugh score & $0.746(0.103-5.422)$ & 0.772 & & \\
\hline CNLC & $0.77(0.464-1.278)$ & 0.312 & & \\
\hline
\end{tabular}

BMI, body mass index; MVI, microvascular invasion; PV-TT, portal vein tumor thrombosis; HBsAg, hepatitis B surface antigen status; TACE, transarterial chemoembolization; AFP, alphafetoprotein; PLT, platelet count; PT, prothrombin time; ALB, albumin; TBIL, total bilirubin; ALT, alanine aminotransferase; AST, aspartate aminotransferase; NEUT, neutrophil count; CNLC, China Liver Cancer Staging; HR, hazard ratio.

modal for predicting DFS and OS was evaluated by calculating $\mathrm{C}$-index values (Table 6). In DFS analysis, the CT+MRI+Clinical model showed the best performance in the training cohort (Cindex $=0.858 ; 95 \% \mathrm{CI}, 0.811-0.905)$, followed by the CT+MRI model (C-index $=0.826 ; 95 \% \mathrm{CI}, 0.767-0.885$ ). The clinical model had the lowest predictive performance of C-index = 0.717 (95\% CI, 0.648-0.786). In the validation cohort, the $\mathrm{CT}+\mathrm{MRI}+\mathrm{Clinical}$ model showed the best performance (Cindex $=0.704 ; 95 \% \mathrm{CI}, 0.563-0.845)$, followed by the clinical model (C-index $=0.657 ; 95 \% \mathrm{CI}, 0.504-0.809)$. The MRI model had the lowest predictive performance of C-index $=0.587(95 \%$ CI, 0.412-0.763).

For analysis of OS, CT+MRI+Clinical had the best predictive performance $(\mathrm{C}$-index $=0.893 ; 95 \% \mathrm{CI}, 0.846-0.940)$ in the training cohort, followed by the CT+MRI model (C-index = 0.865; 95\% CI, 0.810-0.920); the CT model had the lowest predictive performance $(\mathrm{C}$-index $=0.740$; 95\% CI, 0.650-0.830).

In the validation cohort, $\mathrm{CT}+\mathrm{MRI}+\mathrm{Clinical}$ had the best predictive performance $(\mathrm{C}$-index $=0.738$; 95\% CI, $0.575-$ 0.901 ), followed by the clinical model (C-index $=0.705 ; 95 \%$ CI, 0.597-0.803). The MRI model had the lowest predictive performance of C-index $=0.601$ (95\% CI, 0.401-801). The calibration curve showed the high accuracy of the nomograms for predicting DFS and OS both in the training dataset (Figures 3C, D).

\section{DISCUSSION}

Previous studies have developed multimodal imaging models, using radiomics features determined by $\mathrm{MR}$ and $\mathrm{CT}$ to predict tumor prognosis (28). To our knowledge, the present study is the first to evaluate DFS and OS in patients with HCC using a contrastive learning analysis of enhanced CT and MRI sequence data. The main challenges faced by multi-pattern methods are how to judge the confidence of each mode and the correlation between modes, how to reduce the dimension of multi-pattern characteristic information, and how to register multi-pattern data collected asynchronously (13-15). We compared the advantages of multimodal radiomics models for CT and MRI integration.

Radiomics has recently received attention in the field of cancer research because it is a high-throughput method used to extract large numbers of radiomics features from standard medical imaging and can improve medical decisions (29). Radiomics is used to extract quantitative feature data that 
TABLE 3 | Univariate and multivariate analyses of training cohort to identify patient clinical features with prognostic value for OS.

\begin{tabular}{|c|c|c|c|c|}
\hline \multirow[t]{2}{*}{ Variable } & \multicolumn{2}{|c|}{ Univariate analysis } & \multicolumn{2}{|c|}{ Multivariate analysis } \\
\hline & HR (95\% Cl) & $p$-Value & HR (95\% Cl) & $p$-Value \\
\hline Age & 1.018 (0.98-1.057) & 0.351 & & \\
\hline Gender & $0.484(0.215-1.092)$ & 0.081 & & \\
\hline BMl & $0.881(0.798-0.972)$ & $<0.05$ & 0.850 (0.740-0.970) & $<0.05$ \\
\hline Alcohol & $0.92(0.378-2.240)$ & 0.853 & & \\
\hline Liver cirrhosis & $0.952(0.383-2.367)$ & 0.916 & & \\
\hline Histopathological grade & $1.695(0.965-2.977)$ & 0.066 & & \\
\hline Tumor diameter & 1.188 (1.063-1.327) & $<0.05$ & 1.100 (0.910-1.320) & 0.329 \\
\hline Liver capsule invasion & $1.853(0.888-3.867)$ & 0.100 & & \\
\hline Surgical margin & $1.053(0.991-1.120)$ & 0.096 & & \\
\hline Tumor number & 0.947 (0.419-2.139) & 0.895 & & \\
\hline Satellite lesions & $1.136(0.339-3.805)$ & 0.836 & & \\
\hline MVI & 6.935 (2.962-16.239) & $<0.05$ & 5.060 (2.080-12.310) & $<0.05$ \\
\hline PV_TT & $3.87(1.142-13.114)$ & $<0.05$ & $3.190(0.870-11.650)$ & 0.079 \\
\hline $\mathrm{HBV}$ & $0.555(0.212-1.454)$ & 0.231 & & \\
\hline HBsAg & 0.998 (0.994-1.001) & 0.155 & & \\
\hline Surgical approach & 1.267 (0.599-2.680) & 0.535 & & \\
\hline Pos_operation_TACE & $1.305(0.641-2.658)$ & 0.463 & & \\
\hline AFP & 1.000 (0.999-1.000) & 0.136 & & \\
\hline PLT & 0.993 (0.986-1.000) & $<0.05$ & 0.990 (0.990-1.000) & 0.174 \\
\hline PT & 1.003 (0.982-1.024) & 0.812 & & \\
\hline Alb & 1.003 (0.932-1.080) & 0.937 & & \\
\hline TBIL & 0.989 (0.952-1.028) & 0.579 & & \\
\hline ALT & 0.999 (0.995-1.002) & 0.500 & & \\
\hline AST & 0.998 (0.994-1.003) & 0.478 & & \\
\hline NEUT & 1.07 (0.900-1.273) & 0.442 & & \\
\hline Lymphocyte & $0.975(0.936-1.015)$ & 0.219 & & \\
\hline Bleeding_volume & 1.001 (1.001-1.002) & $<0.05$ & 1.000 (1.000-1.010) & $<0.05$ \\
\hline Child-Pugh score & $1.784(0.237-13.428)$ & 0.574 & & \\
\hline CNLC & $1.313(0.787-2.190)$ & 0.298 & & \\
\hline
\end{tabular}

TABLE 4 | Univariate and multivariate analyses of training cohort to identify patient clinical features and Combined_radscore with prognostic value for DFS.

\begin{tabular}{|c|c|c|c|c|}
\hline \multirow[t]{2}{*}{ Variable } & \multicolumn{2}{|c|}{ Univariate analysis } & \multicolumn{2}{|c|}{ Multivariate analysis } \\
\hline & HR $(95 \% \mathrm{Cl})$ & $p$-Value & HR $(95 \%$ Cl) & $p$-Value \\
\hline Tumor diameter & $1.128(1.020-1.247)$ & $<0.05$ & $1.290(0.660-2.520)$ & 0.456 \\
\hline Liver capsule invasion & 1.907 (1.036-3.509) & $<0.05$ & $0.970(0.870-1.080)$ & 0.593 \\
\hline $\mathrm{MVI}$ & $4.338(2.310-8.147)$ & $<0.05$ & $3.090(1.520-6.310)$ & $<0.05$ \\
\hline Radscore & 6.553 (3.975-10.803) & $<0.05$ & $5.600(3.340-9.370)$ & $<0.05$ \\
\hline
\end{tabular}

DFS, disease-free survival; MVI, microvascular invasion; Radscore, radiomics score; HR, hazard ratio.

TABLE 5 | Univariate and multivariate analyses of training cohort to identify patient clinical features and Combined_radscore with prognostic value for OS.

\begin{tabular}{|c|c|c|c|c|}
\hline \multirow[t]{2}{*}{ Variable } & \multicolumn{2}{|c|}{ Univariate analysis } & \multicolumn{2}{|c|}{ Multivariate analysis } \\
\hline & HR (95\% Cl) & $p$-Value & HR (95\% Cl) & $p$-Value \\
\hline BMl & 0.881 (0.798-0.972) & $<0.05$ & 0.970 (0.880-1.060) & 0.480 \\
\hline Tumor diameter & 1.188 (1.063-1.327) & $<0.05$ & $0.840(0.660-1.080)$ & 0.174 \\
\hline MVI & $6.935(2.962-16.239)$ & $<0.05$ & $4.110(1.550-10.87)$ & $<0.05$ \\
\hline$P V_{-} \pi T$ & $3.870(1.142-13.114)$ & $<0.05$ & $2.030(0.510-8.160)$ & 0.318 \\
\hline PLT & $0.993(0.986-1.000)$ & $<0.05$ & $0.990(0.980-1.000)$ & $<0.05$ \\
\hline Bleeding_volume & $1.001(1.001-1.002)$ & $<0.05$ & $1.000(1.000-1.010)$ & $<0.05$ \\
\hline Radscore & 6.959 (3.922-12.349) & $<0.05$ & $7.740(3.560-16.800)$ & $<0.05$ \\
\hline
\end{tabular}

OS, overall survival; BMI, body mass index; MVI, microvascular invasion; PV-TT, portal vein tumor thrombosis; platelet count; Radscore, radiomics score; HR, hazard ratio. 

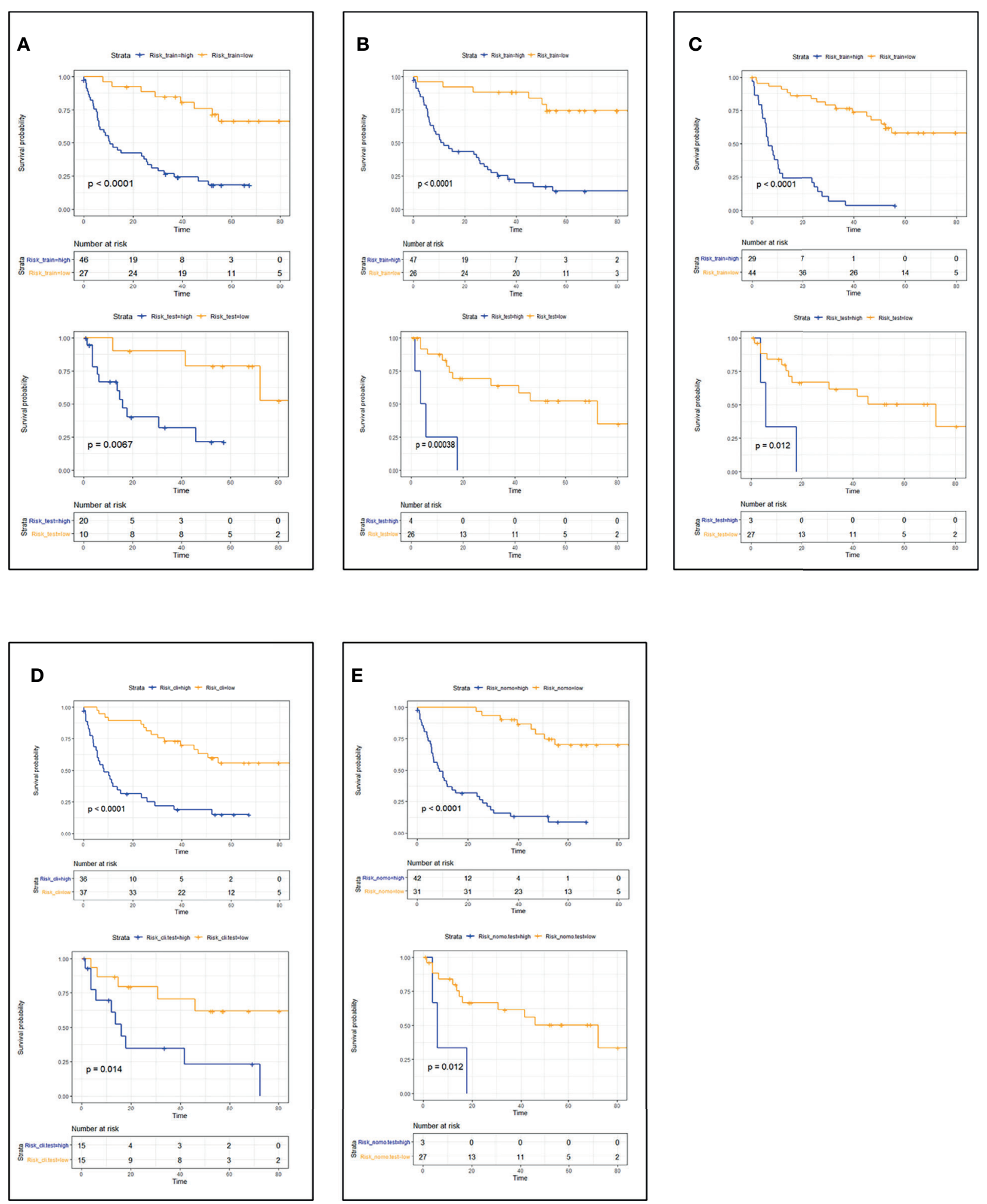

FIGURE 1 | Patient DFS KM curves for each model. (A) CT_DFS; (B) MRI_DFS; (C) CT+MRI_DFS; (D) Clinical_DFS; (E) CT+MRI+Clinical_DFS. p-Values were calculated using the log-rank test. Training cohort curves are shown on the top and validation cohorts on the bottom in each panel. DFS, disease-free survival; KM, Kaplan-Meier. 

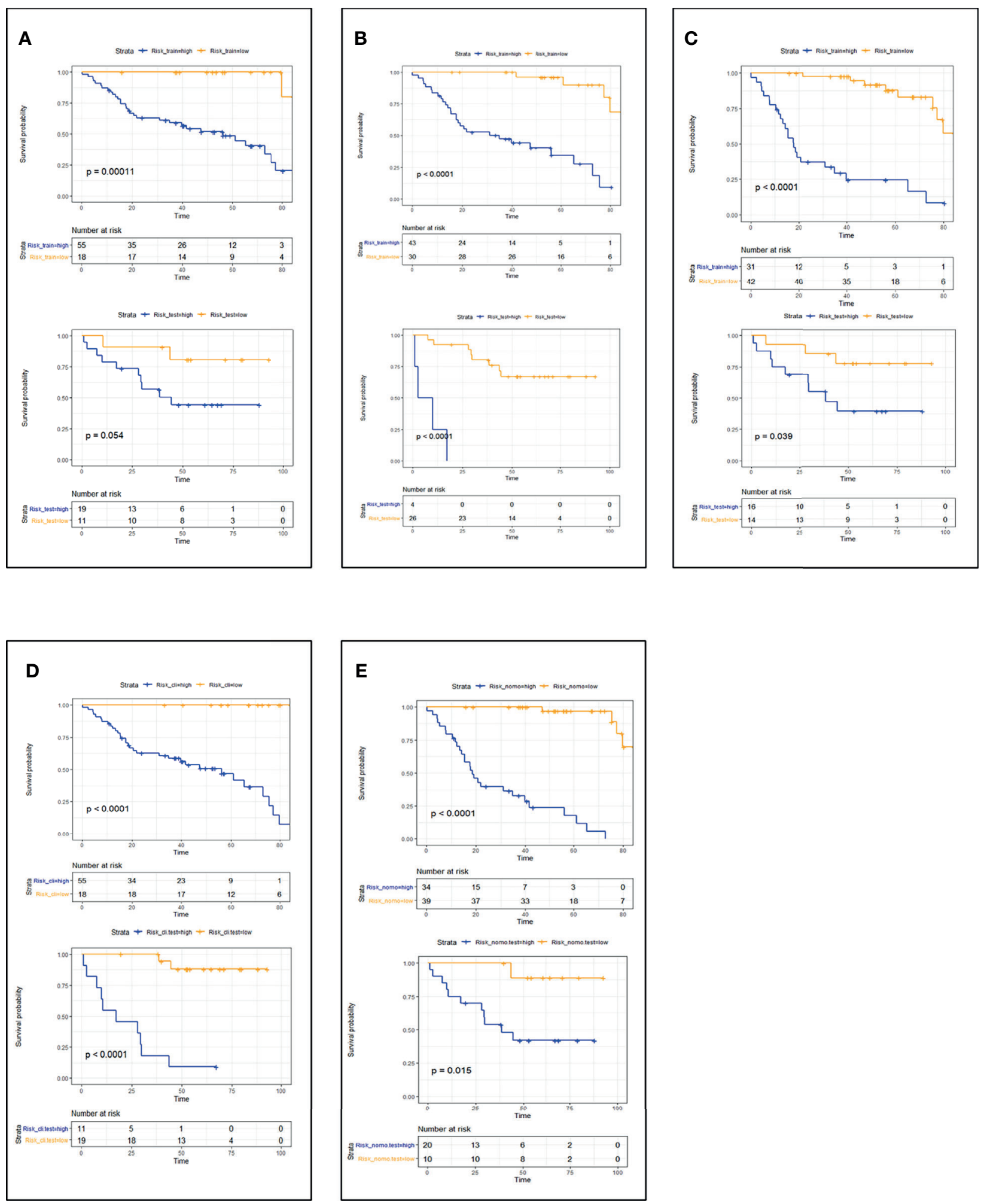

FIGURE 2 | Patient OS KM curves for each model: (A) CT_OS; (B) MRI_OS; (C) CT+MRI_OS; (D) Clinical_OS; (E) CT+MRI+Clinical_OS. p-Values were calculated using the log-rank test. Training cohort curves are shown on the top and validation cohorts on the bottom in each panel. OS, overall survival; KM, Kaplan-Meier. 


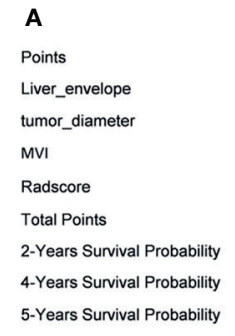

B

Points

BMI

tumor_diameter

MVI

PV-TT

PLT

Bleeding_volume

Radscore

Total Points

2-Years Survival Probability

4-Years Survival Probability

5-Years Survival Probability

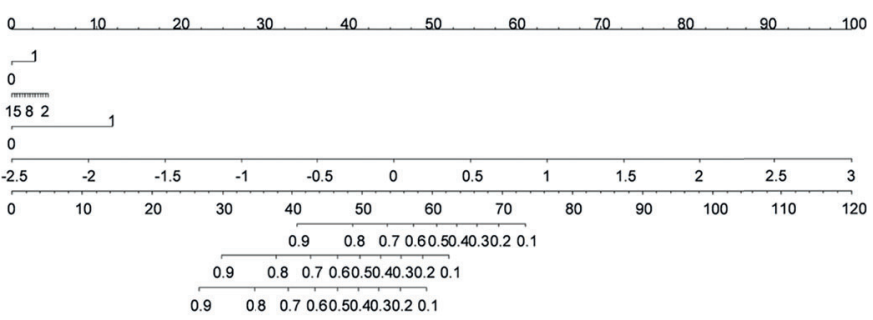

$\begin{array}{lll}0.9 \quad 08 & 0.70 .60 .50 .40 .3020 .1\end{array}$

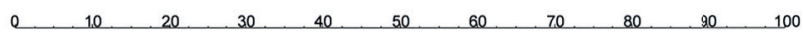

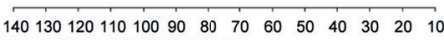

$15 \quad 13 \quad 11 \quad 98276554321$

0

0

$300 \quad 260 \quad 220 \quad 180 \quad 140 \quad 100 \quad 60$

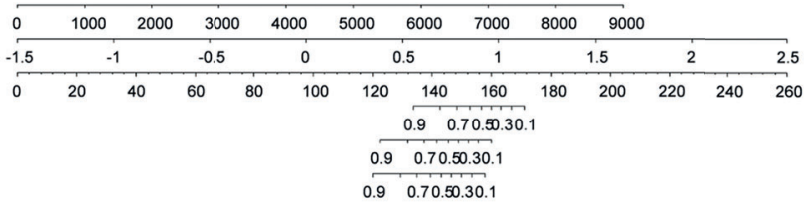

C

D

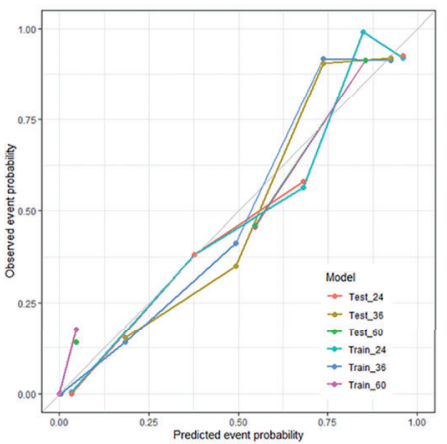

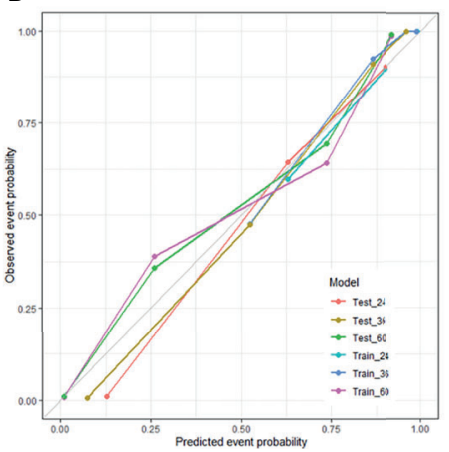

FIGURE 3 | Development of nomograms and calibration curves for DFS and OS in training cohorts. (A) Prognostic nomogram for DFS. (B) The prognostic nomogram for OS. (C) Calibration curves for DFS in the training cohort. (D) Calibration curves for OS in the training cohort. To determine the number of factors associated with the probability of survival, a straight line was drawn to the relevant point on the axis for each patient, and the process was repeated for each variable. Scores for each risk factor were then summarized, with the final sum marked on the overall point axis. DFS and OS estimated using the nomogram are plotted on the x-axis. Observed DFS or OS are plotted on the $y$-axis, and the estimated results are compared with the actual results. The consistency of estimated and observed calibrations for 2-year, 4-year, and 5-year survival results is shown for each model. DFS, disease-free survival; OS, overall survival.

TABLE 6 | The performance of each model in the training and validation cohorts.

\begin{tabular}{|c|c|c|c|c|c|}
\hline \multirow[b]{2}{*}{ Disease-free survival } & \multirow[t]{2}{*}{ Model } & \multicolumn{2}{|c|}{ Training cohort } & \multicolumn{2}{|c|}{ Validation cohort } \\
\hline & & C-index & $95 \% \mathrm{Cl}$ & C-index & $95 \% \mathrm{Cl}$ \\
\hline & CT & 0.742 & $0.668-0.816$ & 0.614 & $0.442-0.786$ \\
\hline & $\mathrm{MRI}$ & 0.772 & $0.705-0.839$ & 0.587 & $0.412-0.763$ \\
\hline & $\mathrm{CT}+\mathrm{MRI}$ & 0.826 & $0.767-0.885$ & 0.653 & $0.490-0.816$ \\
\hline & Clinical & 0.717 & $0.648-0.786$ & 0.657 & $0.504-0.809$ \\
\hline & $\mathrm{CT}+\mathrm{MRI}+$ Clinical & 0.858 & $0.811-0.905$ & 0.704 & $0.563-0.845$ \\
\hline \multirow[t]{5}{*}{ Overall survival } & CT & 0.740 & $0.650-0.830$ & 0.624 & $0.450-0.789$ \\
\hline & $\mathrm{MRI}$ & 0.833 & $0.768-0.898$ & 0.601 & $0.401-0.801$ \\
\hline & $\mathrm{CT}+\mathrm{MRI}$ & 0.865 & $0.810-0.920$ & 0.653 & $0.471-0.835$ \\
\hline & Clinical & 0.802 & $0.714-0.890$ & 0.705 & $0.597-0.803$ \\
\hline & $\mathrm{CT}+\mathrm{MRI}+$ Clinical & 0.893 & $0.846-0.940$ & 0.738 & $0.575-0.901$ \\
\hline
\end{tabular}


reflect information related to tumor heterogeneity, which are not visible to the human eye. Hence, radiomics can provide a noninvasive, low-cost, and reproducible means to capture tumor phenotypes that may be associated with intra-tumor heterogeneity (30). To date, radiomics has been used in research to explore liver tumors, including numerous studies applied to the diagnosis, prognosis, pathological grading, and MVI of liver cancer (31-34). Many previous studies have demonstrated the role of radiomics in survival assessment for patients with different types of cancer, including non-small cell lung, breast, and thyroid cancers (35-37).

We developed a new multimodal radiomics model to compare the value of enhanced CT and MRI sequence data for prognosis prediction in patients with HCC and to compare this with the predictive performance of clinicopathological factors. In this study, we extracted 1,217 features from CT and MR images and finally identified non-zero coefficient features associated with DFS and prognostic features associated with OS by LASSO regression analysis. Specific feature dimension reduction and features screening processes are also shown in the Supplementary Materials. Radscore values were calculated using these features. KM survival analysis methods and log-rank tests were used to evaluate their prognostic value.

In our study, the results of multivariate analyses showed that MVI, Bleeding_volume, and PLT were independent predictors of the prognosis of HCC patients, which was consistent with the results of previous studies (7-10). The CT+MRI+Clinical model was superior to that of a model comprising clinical features alone, CT alone, MRI alone, or CT+MRI combined model, indicating that the multimodal radiomics model approach may have a greater value in predicting DFS and OS of resected HCC. The multimodal model can provide more abundant information.

In addition, for all KM curves of predicting DFS and OS, the low-risk group had significantly higher survival times than the high-risk group $(p<0.05)$, indicating that Radscore was an independent predictor of HCC, and this finding was confirmed in the multivariate Cox proportional risk model $(p<0.05)$ in both DFS and OS. Thus, Radscore improves traditional prognostic ability and represents a potentially effective and promising tool for evaluating the prognosis of patients with HCC. This is consistent with the study by Zhao et al. (38). In a prior study, Zhang et al. (28) established single and multimodal logic models for predicting LVI, with excellent predictive power in training (area under the curve (AUC), 0.884; 95\% CI, 0.8030.964 ) and validation (AUC, 0.876; 95\% CI, 0.721-1.000). Their results are similar to our study, but our model also included clinical factors. Univariate and multivariate Cox analyses were used to select clinical factors into the model to analyze the prognosis, which was more convincing and scientific by comparing the prediction performance of various modes, and it was shown in nomograms. Our Radscore-based nomograms yielded a better discriminative ability than these traditional methods for predicting prognosis in HCC patients.

Zhou et al. $(24,38)$ extracted radiomics features from arterial and portal phase CT images of 215 HCC patients undergoing partial hepatectomy, screened the imaging features through a
LASSO logistic regression model, and constructed a Radscore model. The results showed that inclusion of CT-based radiomics features with routine clinical variables significantly predicted early recurrence ( $\leq 1$ year) postoperatively and that the diagnostic performance of the model combining radiomics and clinical factors was superior to that of the model with clinical features alone for estimating early recurrence. It seems to be obvious that assessing tumorous disease with single modal radiomics information will not be comprehensive. However, the development of methods and strategies for the integration of information of different dimensions is still in its early stages, and combining prediction models, as performed in the current study, might increase their precision and could be extended to other diagnostic indicators. Further research following this scheme is warranted.

This study has several limitations. First, our study was conducted in a single institution. Although all CT and MR images were obtained using a uniform scanner and standardized imaging acquisition sequences, to reduce bias and variance in our results and improve the robustness of the model, further confirmation using patient data from other institutions is needed. Second, the use of manually drawn two-dimensional $\mathrm{ROI}$ is time-consuming and inconvenient for clinical application; hence, the feasibility of automatic segmentation or semisegmentation in radiomics analysis will be the focus of future research. Third, the number of patients in this study is not large because not all HCC patients need to undergo CT and MR in clinical practice. In addition, the cost of conducting CT and MR at the same time is relatively expensive, so there are some obstacles to implementation. Finally, our single-center study primarily included patients who had undergone CT and MR, with a small sample size. We will work with other hospitals to explore the robustness of similar multimodal models in the future.

In conclusion, our results suggest that Radscore is an independent prognostic factor in patients with HCC. Multimodal imaging profiles have great potential to improve individualized assessment of likely prognosis after surgery and may guide the individualized care of patients with HCC.

\section{DATA AVAILABILITY STATEMENT}

The raw data supporting the conclusions of this article will be made available by the authors, without undue reservation.

\section{ETHICS STATEMENT}

The studies involving human participants were reviewed and approved by the Affiliated Hospital of Qingdao University. Written informed consent for participation was not required for this study in accordance with the national legislation and the institutional requirements. 


\section{AUTHOR CONTRIBUTIONS}

$\mathrm{YH}, \mathrm{CZ}, \mathrm{QD}, \mathrm{BH}$, and $\mathrm{XZ}$ contributed to the conception and design. $\mathrm{YH}, \mathrm{BH}, \mathrm{WX}$, and XC organized the database. YG, XH, and $\mathrm{BD}$ managed the patient and provided technical support. $\mathrm{YH}$ wrote the first draft of the manuscript. YH and YG performed the statistical analysis, CZ, QD, and XZ reviewed and revised the manuscript. All authors listed have made a substantial, direct, and intellectual contribution to the work and approved it for publication.

\section{REFERENCES}

1. Bray F, Ferlay J, Soerjomataram I, Siegel RL, Torre LA, Jemal A. Global Cancer Statistics 2018: GLOBOCAN Estimates of Incidence and Mortality Worldwide for 36 Cancers in 185 Countries. CA Cancer J Clin (2018) 68:394424. doi: $10.3322 /$ caac. 21492

2. C. Global Burden of Disease Liver Cancer, Akinyemiju T, Abera S, Ahmed M, Alam N, Alemayohu MA, et al. The Burden of Primary Liver Cancer and Underlying Etiologies From 1990 to 2015 at the Global, Regional, and National Level: Results From the Global Burden of Disease Study 2015. JAMA Oncol (2017) 3:1683-91. doi: 10.1001/jamaoncol.2017.3055

3. Poon RT, Fan ST, Lo CM, Liu CL, Wong J. Long-Term Survival and Pattern of Recurrence After Resection of Small Hepatocellular Carcinoma in Patients With Preserved Liver Function: Implications for a Strategy of Salvage Transplantation. Ann Surg (2002) 235(3):373-82. doi: 10.1097/00000658200203000-00009

4. Shah SA, Cleary SP, Wei AC, Yang I, Taylor BR, Hemming AW, et al. Recurrence After Liver Resection for Hepatocellular Carcinoma: Risk Factors, Treatment, and Outcomes. Surg (2007) 141(3):330-9. doi: 10.1016/j.surg.2006.06.028

5. Lacaze L, Scotte M. Surgical Treatment of Intra Hepatic Recurrence of Hepatocellular Carcinoma. World J Hepatol (2015) 7(13):1755-60. doi: 10.4254/wjh.v7.i13.1755

6. Lau WY, Lai EC. Hepatocellular Carcinoma: Current Management and Recent Advances. Hepatobiliary Pancreat Dis Int (2008) 7:237-57.

7. Li SH, Guo ZX, Xiao CZ, Wei W, Shi M, Chen ZY, et al. Risk Factors for Early and Late Intrahepatic Recurrence in Patients With Single Hepatocellular Carcinoma Without Macrovascular Invasion After Curative Resection. Asian Pac J Cancer Prev (2013) 14:4759-63. doi: 10.7314/apjcp.2013.14.8.4759

8. Zhou YM, Yang JM, Li B, Yin ZF, Xu F, Wang B, et al. Risk Factors for Early Recurrence of Small Hepatocellular Carcinoma After Curative Resection. Hepatobiliary Pancreat Dis Int (2010) 9:33-7.

9. Hirokawa F, Hayashi M, Asakuma M, Shimizu T, Inoue Y, Uchiyama K. Risk Factors and Patterns of Early Recurrence After Curative Hepatectomy for Hepatocellular Carcinoma. Surg Oncol (2016) 25:24-9. doi: 10.1016/ j.suronc.2015.12.002

10. Poon RT, Fan ST, Ng IO, Lo CM, Liu CL, Wong J. Different Risk Factors and Prognosis for Early and Late Intrahepatic Recurrence After Resection of Hepatocellular Carcinoma. Cancer (2000) 89:500-7. doi: 10.1002/1097-0142 (20000801)89:3<500::AID-CNCR4>3.0.CO;2-O

11. Summers RM. Are We at a Crossroads or a Plateau? Radiomics and Machine Learning in Abdominal Oncology Imaging. Abdom Radiol (NY) (2019) 44:1985-9. doi: 10.1007/s00261-018-1613-1

12. Limkin EJ, Sun R, Dercle L, Zacharaki EI, Robert C, Reuze S, et al. Promises and Challenges for the Implementation of Computational Medical Imaging (Radiomics) in Oncology. Ann Oncol (2017) 28:1191-206. doi: 10.1093/ annonc/mdx034

13. Baltrusaitis T, Ahuja C, Morency LP. Multimodal Machine Learning: A Survey and Taxonomy. IEEE Trans Pattern Anal Mach Intell (2019) 41:423-43. doi: 10.1109/TPAMI.2018.2798607

14. Atrey PK, Hossain MA, Saddik AE, Kankanhalli MS. Multimodal Fusion for Multimedia Analysis: A Survey. Multimedia Syst (2010) 16(6):345-79. doi: 10.1007/s00530-010-0182-0

\section{FUNDING}

This work was supported by Clinical Medicine + X (grant number 3756).

\section{SUPPLEMENTARY MATERIAL}

The Supplementary Material for this article can be found online at: https://www.frontiersin.org/articles/10.3389/fonc.2022. 745258/full\#supplementary-material

15. Ramachandram D, Taylor GW. Deep Multimodal Learning: A Survey on Recent Advances and Trends. IEEE Signal Process Mag (2017) 34(6):96-108. doi: 10.1109/MSP.2017.2738401

16. European Association for the Study of the Liver. Electronic address and L. European Association for the Study of the. EASL Clinical Practice Guidelines: Management of Hepatocellular Carcinoma. J Hepatol (2018) 69:182-236. doi: 10.1016/j.jhep.2018.03.019

17. Marrero JA, Kulik LM, Sirlin CB, Zhu AX, Finn RS, Abecassis MM, et al. Diagnosis, Staging, and Management of Hepatocellular Carcinoma: 2018 Practice Guidance by the American Association for the Study of Liver Diseases. Hepatology (2018) 68:723-50. doi: 10.1002/hep.29913

18. Omata M, Cheng AL, Kokudo N, Kudo M, Lee JM, Jia J, et al. Asia-Pacific Clinical Practice Guidelines on the Management of Hepatocellular Carcinoma: A 2017 Update. Hepatol Int (2017) 11:317-70. doi: 10.1007/ s12072-017-9799-9

19. Zech CJ, Ba-Ssalamah A, Berg T, Chandarana H, Chau GY, Grazioli L, et al. Consensus Report From the 8th International Forum for Liver Magnetic Resonance Imaging. Eur Radiol (2020) 30(1):370-82. doi: 10.1007/s00330019-06369-4

20. Vogel A, Cervantes A, Chau I, Daniele B, Llovet JM, Meyer T, et al. ESMO Guidelines Committee. Hepatocellular Carcinoma: ESMO Clinical Practice Guidelines for Diagnosis, Treatment and Follow-Up. Ann Oncol (2018) 29 (Suppl 4):iv238-55. doi: 10.1093/annonc/mdy308

21. Meng XP, Wang YC, Zhou JY, Yu Q, Lu CQ, Xia C, et al. Comparison of MRI and CT for the Prediction of Microvascular Invasion in Solitary Hepatocellular Carcinoma Based on a Non-Radiomics and Radiomics Method: Which Imaging Modality Is Better? J Magn Reson Imaging (2021) 54:526-36. doi: 10.1002/jmri.27575

22. An C, Kim DW, Park YN, Chung YE, Rhee H, Kim MJ. Single Hepatocellular Carcinoma: Preoperative MR Imaging to Predict Early Recurrence After Curative Resection. Radiology (2015) 276:433-43. doi: 10.1148/ radiol.15142394

23. Ahn SJ, Kim JH, Park SJ, Kim ST, Han JK. Hepatocellular Carcinoma: Preoperative Gadoxetic Acid-Enhanced MR Imaging Can Predict Early Recurrence After Curative Resection Using Image Features and Texture Analysis. Abdom Radiol (NY) (2019) 44:539-48. doi: 10.1007/s00261-0181768-9

24. Zhou Y, He L, Huang Y, Chen S, Wu P, Ye W, et al. CT-Based Radiomics Signature: A Potential Biomarker for Preoperative Prediction of Early Recurrence in Hepatocellular Carcinoma. Abdom Radiol (NY) (2017) 42:1695-704. doi: 10.1007/s00261-017-1072-0

25. Zhang B, Tian J, Dong D, Gu D, Dong Y, Zhang L, et al. Radiomics Features of Multiparametric MRI as Novel Prognostic Factors in Advanced Nasopharyngeal Carcinoma. Clin Cancer Res (2017) 23:4259-69. doi: 10.1158/1078-0432

26. Fave X, Zhang L, Yang J, Mackin D, Balter P, Gomez D, et al. Delta-Radiomics Features for the Prediction of Patient Outcomes in Non-Small Cell Lung Cancer. Sci Rep (2017) 7:588. doi: 10.1038/s41598-017-00665-Z

27. Cusumano D, Meijer G, Lenkowicz J, Chiloiro G, Boldrini L, Masciocchi C, et al. A Field Strength Independent MR Radiomics Model to Predict Pathological Complete Response in Locally Advanced Rectal Cancer. Radiol Med (2021) 126:421-9. doi: 10.1007/s11547-020-01266-Z 
28. Zhang Y, He K, Guo Y, Liu X, Yang Q, Zhang C, et al. Novel Multimodal Radiomics Model for Preoperative Prediction of Lymphovascular Invasion in Rectal Cancer. Front Oncol (2020) 10:457. doi: 10.3389/fonc.2020.00457

29. Lambin P, Leijenaar RTH, Deist TM, Peerlings J, de Jong EEC, van Timmeren J, et al. Radiomics: The Bridge Between Medical Imaging and Personalized Medicine. Nat Rev Clin Oncol (2017) 14:749-62. doi: 10.1038/ nrclinonc.2017.141

30. Davnall F, Yip CS, Ljungqvist G, Selmi M, Ng F, Sanghera B, et al. Assessment of Tumor Heterogeneity: An Emerging Imaging Tool for Clinical Practice? Insights Imaging (2012) 3:573-89. doi: 10.1007/s13244-012-0196-6

31. Guo J, Seo Y, Ren S, Hong S, Lee D, Kim S, et al. Diagnostic Performance of Contrast-Enhanced Multidetector Computed Tomography and Gadoxetic Acid Disodium-Enhanced Magnetic Resonance Imaging in Detecting Hepatocellular Carcinoma: Direct Comparison and a Meta-Analysis. Abdom Radiol (NY) (2016) 41:1960-72. doi: 10.1007/s00261-016-0807-7

32. Kim HY, Choi JY, Kim CW, Bae SH, Yoon SK, Lee YJ, et al. Gadolinium Ethoxybenzyl Diethylenetriamine Pentaacetic Acid-Enhanced Magnetic Resonance Imaging Predicts the Histological Grade of Hepatocellular Carcinoma Only in Patients With Child-Pugh Class A Cirrhosis. Liver Transpl (2012) 18:850-7. doi: 10.1002/lt.23426

33. Huppertz A, Haraida S, Kraus A, Zech CJ, Scheidler J, Breuer J, et al. Enhancement of Focal Liver Lesions at Gadoxetic Acid-Enhanced MR Imaging: Correlation With Histopathologic Findings and Spiral CT-Initial Observations. Radiology (2005) 234:468-78. doi: 10.1148/radiol.2342040278

34. Sumie S, Kuromatsu R, Okuda K, Ando E, Takata A, Fukushima N, et al. Microvascular Invasion in Patients With Hepatocellular Carcinoma and Its Predictable Clinicopathological Factors. Ann Surg Oncol (2008) 15:1375-82. doi: 10.1245/s10434-008-9846-9

35. Park H, Lim Y, Ko ES, Cho HH, Lee JE, Han BK, et al. Radiomics Signature on Magnetic Resonance Imaging: Association With Disease-Free Survival in Patients With Invasive Breast Cancer. Clin Cancer Res (2018) 24:4705-14. doi: $10.1158 / 1078-0432$
36. Kirienko M, Cozzi L, Antunovic L, Lozza L, Fogliata A, Voulaz E, et al. Prediction of Disease-Free Survival by the PET/CT Radiomic Signature in Non-Small Cell Lung Cancer Patients Undergoing Surgery. Eur J Nucl Med Mol Imaging (2018) 45:207-17. doi: 10.1007/s00259-017-3837-7

37. Park VY, Han K, Lee E, Kim EK, Moon HJ, Yoon JH, et al. Association Between Radiomics Signature and Disease-Free Survival in Conventional Papillary Thyroid Carcinoma. Sci Rep (2019) 9:4501. doi: 10.1038/s41598018-37748-4

38. Zheng BH, Liu LZ, Zhang ZZ, Shi JY, Dong LQ, Tian LY, et al. Radiomics Score: A Potential Prognostic Imaging Feature for Postoperative Survival of Solitary HCC Patients. BMC Cancer (2018) 18:1148. doi: 10.1186/s12885-0185024-z

Conflict of Interest: YG was employed by GE Healthcare.

The remaining authors declare that the research was conducted in the absence of any commercial or financial relationships that could be construed as a potential conflict of interest.

Publisher's Note: All claims expressed in this article are solely those of the authors and do not necessarily represent those of their affiliated organizations, or those of the publisher, the editors and the reviewers. Any product that may be evaluated in this article, or claim that may be made by its manufacturer, is not guaranteed or endorsed by the publisher.

Copyright (c) $2022 \mathrm{He}, \mathrm{Hu}, \mathrm{Zhu}, \mathrm{Xu}, \mathrm{Ge}, \mathrm{Hao}$, Dong, Chen, Dong and Zhou. This is an open-access article distributed under the terms of the Creative Commons Attribution License (CC BY). The use, distribution or reproduction in other forums is permitted, provided the original author(s) and the copyright owner(s) are credited and that the original publication in this journal is cited, in accordance with accepted academic practice. No use, distribution or reproduction is permitted which does not comply with these terms. 